\title{
Weight control practices, beliefs, self-efficacy, and eating behaviors in college weight class athletes
}

\author{
Ji Seon Lee', Seong Suk $\mathrm{Cho}^{2}$ and Kyung Won $\mathrm{Kim}^{1 \S}$ \\ 'Department of Food and Nutrition, Seoul Women's University, 621 Hwarangro, Nowon-Gu, Seoul 01797, Korea \\ ${ }^{2}$ National Training Center of Korea Olympic Committee, Seoul 01794, Korea
}

BACKGROUND/OBJECTIVES: This study aimed to examine differences in weight control practices, beliefs, self-efficacy, and eating behaviors of weight class athletes according to weight control level.

SUBJECTS/METHODS: Subjects were weight class athletes from colleges in Gyeong-gi Province. Subjects $(n=182)$ responded to a questionnaire assessing study variables by self-report, and data on 151 athletes were used for statistical analysis. Subjects were categorized into High vs. Normal Weight Loss (HWL, NWL) groups depending on weight control level. Data were analyzed using t-test, ANCOVA, $\mathrm{X}^{2}$-test, and multiple logistic regressions.

RESULTS: Seventy-three percent of subjects were in the HWL group. The two groups showed significant differences in weight control practices such as frequency $(P<0.01)$, duration and magnitude of weight loss, methods, and satisfaction with weight control $(P<0.001)$. Multiple logistic regression showed that self-efficacy (OR: $0.846,95 \% \mathrm{Cl}: 0.730,0.980)$, eating behaviors during training period (OR: $1.285,95 \% \mathrm{Cl}: 1.112,1.485)$, and eating behaviors during the weight control period (OR: $0.731,95 \% \mathrm{Cl}$ : $0.620,0.863)$ were associated with weight control level. Compared to NWL athletes, HWL athletes agreed more strongly on the disadvantages of rapid weight loss $(P<0.05-P<0.01)$, perceived less confidence in controlling overeating after matches $(P<0.001)$, and making weight within their weight class $(P<0.05)$. HWL athletes showed more inappropriate eating behaviors than NWL athletes, especially during the weight control period $(P<0.05-P<0.001)$.

CONCLUSIONS: Self-efficacy was lower and eating behaviors during pre-competition period were more inadequate in HWL athletes. Education programs should include strategies to help athletes apply appropriate methods for weight control, increase self-efficacy, and adopt desirable eating behaviors.

Nutrition Research and Practice 2020;14(1):45-54; https://doi.org/10.4162/nrp.2020.14.1.45; pISSN 1976-1457 elSSN 2005-6168

Keywords: Weight class athlete, weight control, belief, self-efficacy, eating behavior

\section{INTRODUCTION}

Adequate nutrition for athletes is important to maintain physical condition and achieve optimal athletic performance [1]. Careful nutritional management is required for weight class athletes who have to control their weight before competition [2]. Athletes such as taekwondo, judo, boxing, and wrestling athletes are classified based on body weight. This makes competitions more compatible with respect to body size, strength, and agility $[3,4]$. Many weight class athletes, however, practice short-term weight loss to compete at a lower weight class for the purpose of competing with lighter or weaker opponents [5,6].

Despite the importance of adequate diet for weight class athletes, studies have shown that their diets did not meet recommended nutrient intakes [7-10]. In a study on adolescent taekwondo athletes, nutrient intakes, including energy, thiamin, riboflavin, vitamin $\mathrm{C}$, folate, and calcium intakes both before and after competition as well as at ordinary times, were lower than recommended dietary intakes [7]. Similarly, college taekwondo athletes showed insufficient intakes of energy, calcium, and riboflavin over a 12-week pre-competition period [8]. Dietary management for weight class athletes is important according to general training period, before and after competition, and recovery period. However, it has been reported that many weight class athletes do not recognize the importance of nutrition and try to lose weight rapidly before competition $[6,8,11,12]$.

Appropriate weight control can be done through nutritional management, increasing physical activity, and exercise. Professionals recommend that $5 \%$ or less loss of body weight is appropriate in the case of weight control athletes [4,13]. As for methods of weight control, weight class athletes fast, restrict fluid intake, train in plastic suits and saunas, as well as diet and exercise $[5,6,8,11]$. In addition, many weight class athletes try to lose weight in a short time period $[2,12]$. Weight loss

\footnotetext{
This work was supported by a research grant from Seoul Women's University (2017).

${ }^{\S}$ Corresponding Author: Kyung Won Kim, Tel. 82-2-970-5647, Fax. 82-2-976-4049, Email. kwkim@swu.ac.kr

Received: August 20, 2019, Revised: September 6, 2019, Accepted: October 14, 2019

This is an Open Access article distributed under the terms of the Creative Commons Attribution Non-Commercial License (http://creativecommons.org/licenses/by-nc/3.0/) which permits unrestricted non-commercial use, distribution, and reproduction in any medium, provided the original work is properly cited.
} 
in a short time (e.g., more than $5 \%$ loss of body weight within 1 week) has adverse effects on aerobic endurance, body function (e.g., cardiovascular, respiratory, and renal function), emotions, and overall sport performance $[4,14,15]$.

In order to help athletes adopt desirable weight control practices, factors related to weight control need to be examined. Psychosocial theories suggest ways of examining factors related to health behaviors [16-18]. Constructs such as beliefs and self-efficacy regarding behavior, have been suggested in the Social Cognitive Theory (SCT) and the Theory of Planned Behavior (TPB). Beliefs (outcome expectations in the $\mathrm{SCT}$, behavioral beliefs in the TPB) are the perceived advantages or disadvantages of performing a certain behavior. Self-efficacy indicates the perceived ability to perform the behavior and is known as an important predictor of health behaviors [16-18]. Studies on athletes reported that self-efficacy positively influences exercise adherence $[19,20]$.

Previous nutritional studies on weight class athletes have mostly focused on weight control status $[8,21]$ and nutrient intakes during different time periods $[7,8,10]$. There have been few studies, however, that have examined weight control practices and psychosocial factors (i.e., beliefs, self-efficacy) in college weight class athletes. This study was conducted to examine weight control practices as well as to investigate whether or not weight control practices, beliefs regarding rapid weight loss, self-efficacy for eating behaviors and weight control, eating behaviors during training, and weight control period differ according to weight control level in college weight class athletes. This study will provide baseline information for developing education programs for desirable weight control in college weight class athletes.

\section{SUBJECTS AND METHODS}

\section{Subjects}

Subjects for this study were weight class athletes recruited from two colleges in Gyeong-gi province, Korea. The colleges contacted for the study had weight class athletes in taekwondo, judo, and boxing. After getting permission from coaches, investigators explained the study to the athletes, and those who were willing to participate in the survey provided written informed consent. Subjects were also informed that they could withdraw from the study if they were not willing to respond to the survey questionnaire. Data were collected by self-report from 182 weight class athletes. Excluding the data on athletes who were not weight-classed (e.g., taekwondo poomsae) or did not control their weight as well as those who responded incompletely to major study variables (i.e., weight control practices), data from 151 athletes (completion rate: 83.0\%) were used for the statistical analysis. The survey was done during 2016. This study was approved by the Institutional Review Board of Seoul Women's University (IRB-2015A-37).

\section{Measurements}

The survey questionnaire was developed by literature review regarding weight control in weight class athletes [5,6,11,21-24]. The questionnaire draft was revised based on comments from sports nutrition professionals. The survey questionnaire included items measuring general characteristics, weight control practices, beliefs regarding rapid weight control, self-efficacy for eating behaviors and weight control, eating behaviors during training period and weight control period (1 week before competition), and dietary intakes during different time periods. Results on dietary intakes in male athletes were previously reported [25].

General characteristics included items such as age, gender, height, weight, grade, sports type, and age of starting sports $[21,22]$. Weight control practices were examined by items, including starting age of weight control, frequency of weight control per year, duration of weight reduction before competition, magnitude of weight reduction per trial, maximum weight reduction, appropriate degree of weight reduction, education experience regarding weight control, methods used for weight control, and satisfaction with weight control methods $[5,6,11$, $21,22,26]$.

Items for assessment of beliefs regarding rapid weight control were developed based on the literature $[27,28]$. These included advantages (e.g., playing a match in desired weight class, increased speed/flexibility, and increased concentration for sports during training) and disadvantages of weight control in a short time (e.g., difficulty in eating a variety of foods, reduced digestibility, deteriorating cognitive ability and situational judgment, increased risk of injury, and making me unhealthy). These items were rated on a 5-point scale ranging from 'strongly disagree' (1) to 'strongly agree' (5) to indicate the strength of these beliefs. Total score for beliefs was defined as the summated score of eight beliefs, while reversely coding the score on the disadvantages of weight control. The higher total score indicated having more favorable beliefs toward rapid weight control. The Cronbach's alpha for beliefs regarding rapid weight control was 0.76 .

Items for self-efficacy were made to measure the perceived confidence in performing specific eating behaviors and weight control using appropriate methods, based on the literature $[23,29,30]$. Five items were used to measure self-efficacy, including perceived confidence in 'eating low calorie foods instead of high calorie foods', 'food selection for snacks', 'resisting eating high calorie foods in tempting situations', 'controlling excessive overeating/binge eating after matches', and 'making weight within weight class by gradual dieting and exercise'. These items were measured on a 5-point scale ranging from 'very difficult' (1) to 'very easy' (5). Total score for self-efficacy was the summated score of five self-efficacy items. The higher total score for self-efficacy indicated having more confidence in performing eating behaviors related to weight control. The Cronbach's alpha for self-efficacy was 0.71 .

Items for eating behaviors consisted of 16 items [24,31,32]. These included four items on general eating behaviors (e.g. eating regularly, eating an adequate amount of meals, eating a variety of foods, and having breakfast), seven items on eating foods in foods groups (e.g., grains, protein foods, vegetables, dairy products, etc.), and five items on undesirable eating behaviors (e.g., eating foods high in fat, sodium, sugar, processed/instant foods, etc.). Eating behaviors were measured during both the general training period and weight control period ( 1 week before competition). These items were measured by asking subjects to check on the categories (0-2 days, 3-5 
days, 6-7 days a week) that applied to them. To calculate the total score for eating behaviors, each item was coded from 1 (0-2 days a week) to 3 (6-7 days a week). Total score for eating behaviors was the summated score of 16 eating behaviors, while reversely coding the score on undesirable eating behaviors. The higher total score for eating behaviors indicated having more desirable eating behaviors. The Cronbach's alpha for eating behaviors was 0.57 during the training period and 0.53 during the weight control period.

\section{Statistical analysis}

Data were analyzed using SPSS (PASW Statistics 21.0; SPSS Inc., Chicago, IL, USA). Subjects were categorized into two groups by weight control level, 'High Weight Loss (HWL, >5\% loss of body weight)' and 'Normal Weight Loss (NWL, $\leq 5 \%$ loss of body weight)'. Group categorization was performed based on the literature, which has shown that the appropriate level of weight control for athletes is about $5 \%$ or less of body weight $[1,4,13]$ and that rapid weight loss ( $>5 \%$ loss of body weight in a short time) adversely affects normal growth, aerobic and anaerobic exercise capacity, competitive performance, and psychological function $[4,13,14,33]$. T-test, analysis of covariance (ANCOVA), or $\mathrm{X}^{2}$-test was used to examine differences in weight control practices, beliefs regarding rapid weight control, self-efficacy, eating behaviors during training period, and eating behaviors during weight control period by weight control level. Multiple logistic regressions were employed to examine the association of factors (i.e., beliefs, self-efficacy, and eating behaviors) to weight control while adjusting for demographic covariates. Multiple logistic regressions were performed at two different levels: total score and subscales of beliefs, self-efficacy, and eating behaviors. Factor analysis was performed to find the subscales. Statistical significance was examined at $P<0.05$.

\section{RESULTS}

General characteristics of subjects by weight control level

Mean age of subjects was 19.8 years, and $72.8 \%$ were male athletes (Table 1). About $72.8 \%$ (110 athletes) were categorized into the HWL group, whereas $27.2 \%$ (41 athletes) were in the NWL group. The mean height, weight, and body mass index (BMI) of male subjects were $178.0 \mathrm{~cm}, 74.3 \mathrm{~kg}$, and $23.4 \mathrm{~kg} / \mathrm{m}^{2}$, respectively. The mean weight $(P<0.01)$ and BMl $(P<0.01)$ of male subjects were significantly lower in the HWL group than the NWL group. The mean height, weight, and BMI of female subjects were $168.3 \mathrm{~cm}, 63.1 \mathrm{~kg}, 22.3 \mathrm{~kg} / \mathrm{m}^{2}$, respectively, and

Table 1. General characteristics of subjects by weight control level

\begin{tabular}{|c|c|c|c|c|}
\hline \multirow[b]{2}{*}{ Variables } & \multirow[b]{2}{*}{$\begin{array}{c}\text { Total } \\
(n=151)\end{array}$} & \multicolumn{2}{|c|}{${\text { Weight } \text { control }^{1)}}$} & \multirow[b]{2}{*}{$t$ or $x^{24)}$} \\
\hline & & $\begin{array}{c}\text { NWL } \\
(n=41)\end{array}$ & $\begin{array}{c}\text { HWL } \\
(n=110)\end{array}$ & \\
\hline Age (yrs) & $19.8 \pm 1.2^{2)}$ & $20.1 \pm 1.2$ & $19.6 \pm 1.2$ & $2.4^{*}$ \\
\hline \multicolumn{5}{|l|}{ Gender } \\
\hline Male & $110(72.8)^{3)}$ & $30(73.2)$ & $80(72.7)$ & 0.0 \\
\hline Female & $41(27.2)$ & $11(26.8)$ & $30(27.3)$ & \\
\hline \multicolumn{5}{|l|}{ Height (cm) } \\
\hline Male & $178.0 \pm 6.7$ & $179.3 \pm 7.0$ & $177.6 \pm 6.6$ & 1.2 \\
\hline Female & $168.3 \pm 7.0$ & $170.9 \pm 7.1$ & $167.4 \pm 6.8$ & 1.5 \\
\hline \multicolumn{5}{|l|}{ Weight (kg) } \\
\hline Male & $74.3 \pm 11.7$ & $80.5 \pm 10.2$ & $72.0 \pm 11.4$ & $3.6^{* *}$ \\
\hline Female & $63.1 \pm 7.9$ & $66.8 \pm 5.7$ & $61.8 \pm 8.2$ & 1.9 \\
\hline \multicolumn{5}{|l|}{ Body Mass Index $\left(\mathrm{kg} / \mathrm{m}^{2}\right)$} \\
\hline Male & $23.4 \pm 3.2$ & $25.0 \pm 2.8$ & $22.8 \pm 3.1$ & $3.4^{* *}$ \\
\hline Female & $22.3 \pm 2.2$ & $22.9 \pm 2.1$ & $22.0 \pm 2.2$ & 1.2 \\
\hline \multicolumn{5}{|l|}{ Grade } \\
\hline Freshmen & 51 (33.8) & $11(26.8)$ & $40(36.4)$ & 4.8 \\
\hline Sophomores & $37(24.5)$ & $8(19.5)$ & $29(26.4)$ & \\
\hline Juniors & $32(21.2)$ & $9(22.0)$ & $23(20.9)$ & \\
\hline Seniors & $31(20.5)$ & $13(31.7)$ & $18(16.4)$ & \\
\hline \multicolumn{5}{|l|}{ Sports type } \\
\hline Taekwondo & 93 (61.6) & $20(48.8)$ & $73(66.4)$ & $6.5^{*}$ \\
\hline Boxing & $18(11.9)$ & $4(9.8)$ & $14(12.7)$ & \\
\hline Judo & $40(26.5)$ & $17(41.5)$ & $23(20.9)$ & \\
\hline Age of starting sports (yrs) & $12.3 \pm 2.6$ & $12.8 \pm 2.7$ & $12.1 \pm 2.6$ & 1.5 \\
\hline Participating in competitions during the last 12 months (times) & $5.3 \pm 2.7$ & $5.5 \pm 2.8$ & $5.3 \pm 2.7$ & 0.6 \\
\hline Winning competitions during the last 12 months (times) & $2.4 \pm 2.4$ & $2.4 \pm 2.4$ & $2.4 \pm 2.4$ & 0.0 \\
\hline
\end{tabular}

\footnotetext{
* $P<0.05$, ** $P<0.01$

1) NWL, $\leq 5 \%$ loss of body weight; HWL, $>5 \%$ loss of body weight.

2) Mean $\pm \mathrm{SD}$

3) $\mathrm{N}(\%)$

4) $t$ value by $\mathrm{t}$-test or $\chi^{2}$ value by $\chi^{2}$-test
} 
these were not significantly different by weight control level.

About $61.6 \%$ of respondents were taekwondo athletes, $26.5 \%$ were judo athletes, and $11.9 \%$ were boxers; distribution of sports type was significantly different by weight control level $(P<0.05)$. Subjects started their sport at 12.3 years of age, on average. They competed 5.3 times and won the competitions 2.4 times during the last 12 months, which were not statistically different by weight control level (Table 1).

Weight control practices of subjects by weight control level

Subjects started weight control at 15.7 years of age, on average (Table 2). After considering demographic covariates that were significantly related to weight control level in Table 1 , it was shown that subjects in the HWL group practiced weight control more frequently than the NWL group $(P<0.01)$. Duration of weight reduction before competition $(P<0.001)$, magnitude of weight reduction per weight control trial (mean: $5.9 \mathrm{~kg}$ vs. $2.7 \mathrm{~kg}, P<0.001)$ and maximum weight reduction per trial (mean: $8.0 \mathrm{~kg}$ vs. $6.1 \mathrm{~kg}, P<0.001$ ), and appropriate degree of weight reduction $(P<0.001)$ were also significantly higher in the HWL than the NWL group. Only $19.2 \%$ of subjects received education regarding weight control (Table 2).

Subjects used 'decreasing food intake' (92.1\%), 'increasing activity/training' (80.1\%), 'wearing plastic suits' (73.5\%), and 'fasting' (72.8\%) to lose weight (Table 2). Significantly higher percentages of subjects in the HWL than the NWL group used inappropriate methods such as 'fasting' $(P<0.001)$, 'restricting fluid intake' $(P<0.01)$, 'wearing plastic suits' $(P<0.05)$, 'sauna' $(P<0.05)$, and 'diuretics/laxatives' $(P<0.05)$. About $34.5 \%$ of the

Table 2. Weight control practices of subjects by weight control level

\begin{tabular}{|c|c|c|c|c|}
\hline \multirow[b]{2}{*}{ Variables } & \multirow[b]{2}{*}{$\begin{array}{c}\text { Total } \\
(n=151)\end{array}$} & \multicolumn{2}{|c|}{ 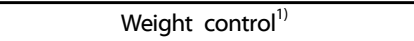 } & \multirow[b]{2}{*}{$x^{2}$ or $F^{4}$} \\
\hline & & $\begin{array}{c}\text { NWL } \\
(n=41)\end{array}$ & $\begin{array}{c}\text { HWL } \\
(n=110)\end{array}$ & \\
\hline Starting age of weight control & $15.7 \pm 2.5^{2)}$ & $16.3 \pm 2.8$ & $15.5 \pm 2.4$ & 2.4 \\
\hline Number of weight control per year & $5.1 \pm 2.2$ & $4.3 \pm 2.2$ & $5.4 \pm 2.2$ & $8.7^{* *}$ \\
\hline Duration of weight reduction before competition (days) & $11.1 \pm 5.7$ & $7.3 \pm 5.1$ & $12.6 \pm 5.3$ & $20.8^{* * *}$ \\
\hline Weight reduction per trial $(\mathrm{kg})$ & $5.0 \pm 2.0$ & $2.7 \pm 0.9$ & $5.9 \pm 1.6$ & $140.7^{* * *}$ \\
\hline Maximum weight reduction $(\mathrm{kg})$ & $7.5 \pm 2.6$ & $6.1 \pm 2.8$ & $8.0 \pm 2.4$ & $25.5^{* * *}$ \\
\hline Appropriate degree of weight reduction $(\mathrm{kg})$ & $3.4 \pm 1.0$ & $3.0 \pm 0.9$ & $3.6 \pm 1.0$ & $17.1^{* * *}$ \\
\hline \multicolumn{5}{|l|}{ Received education regarding weight control } \\
\hline Yes & $29(19.2)^{3)}$ & $6(14.6)$ & $23(20.9)$ & 0.8 \\
\hline No & $122(80.8)$ & $35(85.4)$ & $87(79.1)$ & \\
\hline \multicolumn{5}{|l|}{ Methods used for weight control } \\
\hline \multirow[t]{2}{*}{ Decreasing food intake } & $139(92.1)$ & $35(85.4)$ & $104(94.5)$ & 3.4 \\
\hline & $12(7.9)$ & $6(14.6)$ & $6(5.5)$ & \\
\hline \multirow[t]{2}{*}{ Fasting } & $110(72.8)$ & $17(41.5)$ & $93(84.5)$ & $28.0^{* * *}$ \\
\hline & $41(27.2)$ & $24(58.5)$ & $17(15.5)$ & \\
\hline \multirow[t]{2}{*}{ Restricting fluid intake } & $86(57.0)$ & $14(34.1)$ & $72(65.5)$ & $11.9^{* *}$ \\
\hline & $65(43.0)$ & $27(65.9)$ & $38(34.5)$ & \\
\hline \multirow[t]{2}{*}{ Increasing activity/training } & $121(80.1)$ & $32(78.0)$ & $89(80.9)$ & 0.2 \\
\hline & $30(19.9)$ & $9(22.0)$ & $21(19.1)$ & \\
\hline \multirow[t]{2}{*}{ Sauna } & $86(57.0)$ & $18(43.9)$ & $68(61.8)$ & $3.9^{*}$ \\
\hline & $65(43.0)$ & $23(56.1)$ & $42(38.2)$ & \\
\hline \multirow[t]{2}{*}{ Plastic suits } & $111(73.5)$ & $25(61.0)$ & $86(78.2)$ & $4.5^{*}$ \\
\hline & $40(26.5)$ & $16(39.0)$ & $24(21.8)$ & \\
\hline \multirow[t]{2}{*}{ Diuretics/Laxatives } & $14(9.3)$ & $0(0.0)$ & $14(12.7)$ & $5.8^{*}$ \\
\hline & $137(90.7)$ & $41(100)$ & $96(87.3)$ & \\
\hline \multirow[t]{2}{*}{ Vomiting } & $6(4.0)$ & $0(0.0)$ & $6(5.5)$ & 2.3 \\
\hline & $145(96.0)$ & $41(100)$ & $104(94.5)$ & \\
\hline \multicolumn{5}{|l|}{ Satisfaction with the weight control methods } \\
\hline Not satisfied at all & $16(10.6)$ & $1(2.4)$ & 15 (13.6) & $31.6^{* * *}$ \\
\hline Not satisfied & $25(16.6)$ & $2(4.9)$ & $23(20.9)$ & \\
\hline Neither unsatisfied nor satisfied & $77(51.0)$ & $17(41.5)$ & $60(54.5)$ & \\
\hline Satisfied & $26(17.2)$ & $17(41.5)$ & $9(8.2)$ & \\
\hline Satisfied very much & $7(4.6)$ & $4(9.8)$ & $3(2.7)$ & \\
\hline
\end{tabular}

* $P<0.05,{ }^{* \star} P<0.01,{ }^{* \star *} P<0.001$

1) $\mathrm{NWL}, \leq 5 \%$ loss of body weight; HWL, $>5 \%$ loss of body weight.

2) Mean $\pm S D$

3) $\mathrm{N}(\%)$

4) $\chi^{2}$ value by $\chi^{2}$-test or $\mathrm{F}$ value by ANCOVA with covariates of age, BMl and sports type. 
HWL group, compared to $7.3 \%$ of the NWL group, were 'not satisfied at all' or 'not satisfied' with the weight control methods that they used $(P<0.001)$.

Beliefs regarding rapid weight control by weight control level

Total score for beliefs regarding rapid weight control (possible score: 8-40) was 23.2, which was 58.0 out of 100 (Table 3). Total score for beliefs regarding rapid weight control was significantly lower in the HWL group than the NWL group $(P<0.05)$.

When examined by each belief item using ANCOVA, three out of the eight beliefs were significantly related to weight control level. Subjects in the HWL compared to the NWL group, agreed more strongly on the disadvantages of rapid weight loss, including 'difficulty in eating a variety of foods' $(P<0.01)$, 'making me unhealthy' $(P<0.01)$, and 'deteriorating cognitive ability and situational judgment' $(P<0.05)$. In contrast, none of the beliefs regarding advantages of rapid weight loss was significantly different between the HWL and NWL groups (Table 3).

Self-efficacy regarding eating behaviors and weight control by weight control level

Total score for self-efficacy (possible score: 5-25) was 14.9, which was 59.6 out of 100 (Table 4). The ANCOVA results show that the total score for self-efficacy was significantly lower in the HWL group than the NWL group $(P<0.01)$. Two out of five self-efficacy items differed significantly by weight control level after considering the covariates of age, BMI, and sports type. Subjects in the HWL compared to the NWL group, perceived less self-efficacy in 'controlling overeating/binge eating after matches' $(P<0.001)$, and 'making weight within the desired weight class through gradual dieting and exercise' $(P<0.05)$

Table 3. Beliefs regarding rapid weight control of subjects by weight control level

\begin{tabular}{|c|c|c|c|c|}
\hline \multirow[b]{2}{*}{ Variables } & \multirow[b]{2}{*}{$\begin{array}{c}\text { Total } \\
(\mathrm{n}=151)\end{array}$} & \multicolumn{2}{|c|}{ 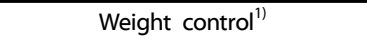 } & \multirow[b]{2}{*}{$\mathrm{F}^{5)}$} \\
\hline & & $\begin{array}{c}\text { NWL } \\
(n=41)\end{array}$ & $\begin{array}{c}\text { HWL } \\
(n=110)\end{array}$ & \\
\hline \multicolumn{5}{|l|}{ Beliefs regarding advantages of rapid weight control } \\
\hline 1. I can play a match in my desired weight class. ${ }^{2)}$ & $3.6 \pm 1.0^{4)}$ & $3.8 \pm 0.9$ & $3.6 \pm 1.0$ & 0.5 \\
\hline 2. It will help to increase speed and flexibility more than ever. & $3.3 \pm 0.9$ & $3.2 \pm 0.8$ & $3.4 \pm 0.9$ & 0.5 \\
\hline 3. It will help to increase concentration for sports during training. & $2.8 \pm 1.0$ & $3.0 \pm 0.8$ & $2.8 \pm 1.0$ & 0.5 \\
\hline \multicolumn{5}{|l|}{ Beliefs regarding disadvantages of rapid weight control } \\
\hline 4. It will be difficult to eat a wide variety of nutritious foods. & $3.8 \pm 1.0$ & $3.4 \pm 1.1$ & $4.0 \pm 0.9$ & $9.9^{* *}$ \\
\hline 5. It will reduce digestibility due to decreased eating amount. & $3.3 \pm 1.0$ & $3.0 \pm 1.0$ & $3.4 \pm 1.0$ & 1.5 \\
\hline 6. It will deteriorate cognitive ability and situational judgment. & $2.8 \pm 1.0$ & $2.5 \pm 1.0$ & $3.0 \pm 1.0$ & $5.9^{*}$ \\
\hline 7. It will increase risk of injury. & $3.0 \pm 1.0$ & $2.7 \pm 1.1$ & $3.1 \pm 1.0$ & 3.1 \\
\hline 8. It will make me unhealthy. & $3.7 \pm 1.0$ & $3.3 \pm 1.2$ & $3.8 \pm 0.9$ & $8.5^{* *}$ \\
\hline Total score of beliefs ${ }^{3}$ & $23.2 \pm 4.8$ & $25.1 \pm 4.3$ & $22.5 \pm 4.8$ & $6.4^{*}$ \\
\hline
\end{tabular}

* $P<0.05,{ }^{* *} P<0.01$

1) $\mathrm{NWL}, \leq 5 \%$ loss of body weight; HWL, $>5 \%$ loss of body weight.

2) Each item was measured by 5 -point scales from 1 (strongly disagree) to 5 (strongly agree). The higher score indicates that subjects agreed more strongly with each belief statement.

3) Possible score: 8-40. To calculate the total score, the items from 4 to 8 were scored reversely.

4) Mean $\pm \mathrm{SD}$

5) $\mathrm{F}$ value by ANCOVA with covariates of age, BMl and sports type

Table 4. Self-efficacy of subjects by weight control level

\begin{tabular}{|c|c|c|c|c|}
\hline \multirow[b]{2}{*}{ Variables } & \multirow{2}{*}{$\begin{array}{c}\text { Total } \\
(\mathrm{n}=151)\end{array}$} & \multicolumn{2}{|c|}{ Weight control ${ }^{1)}$} & \multirow[b]{2}{*}{$\mathrm{F}^{5)}$} \\
\hline & & $\begin{array}{c}\text { NWL } \\
(n=41)\end{array}$ & $\begin{array}{c}\text { HWL } \\
(n=110)\end{array}$ & \\
\hline $\begin{array}{l}\text { 1. I can eat low calorie foods (e.g., fish, chicken breast, etc.) instead of high } \\
\text { calorie foods (e.g., fast food, fried chicken). }{ }^{2}\end{array}$ & $3.0 \pm 1.0^{4)}$ & $3.0 \pm 1.1$ & $3.0 \pm 0.9$ & 1.1 \\
\hline 2. I can select water or fruits instead of soda, bread, and cookies as snacks. & $3.5 \pm 1.0$ & $3.7 \pm 0.9$ & $3.4 \pm 1.0$ & 2.4 \\
\hline $\begin{array}{l}\text { 3. I can resist eating high calorie foods (e.g., fried foods, ice cream, etc.) when } \\
\text { these foods are in front of me. }\end{array}$ & $2.8 \pm 1.1$ & $2.9 \pm 0.8$ & $2.8 \pm 1.1$ & 0.3 \\
\hline 4. I can control excessive overeating and binge eating after matches. & $2.6 \pm 1.1$ & $3.2 \pm 1.0$ & $2.4 \pm 1.0$ & $17.2^{* * *}$ \\
\hline $\begin{array}{l}\text { 5. I can make weight within weight class that I want through gradual dieting } \\
\text { and exercise. }\end{array}$ & $3.1 \pm 0.9$ & $3.4 \pm 0.9$ & $2.9 \pm 0.9$ & $5.3^{*}$ \\
\hline Total score of self-efficacy ${ }^{3)}$ & $14.9 \pm 3.4$ & $16.2 \pm 3.1$ & $14.5 \pm 3.3$ & $7.7^{* *}$ \\
\hline
\end{tabular}

Total score of self-efficacy ${ }^{3}$

* $P<0.05,{ }^{* *} P<0.01,{ }^{* * *} P<0.001$

1) $\mathrm{NWL}, \leq 5 \%$ loss of body weight; HWL, $>5 \%$ loss of body weight.

2) Each item was measured by 5 -point scales from 1 (very difficult) to 5 (very easy). The higher score means the higher self-efficacy regarding eating behavior and weight control.

3) Possible score: $5-25$

4) Mean $\pm \mathrm{SD}$

${ }^{5)} \mathrm{F}$ value by ANCOVA with covariates of age, BMl and sports type. 
Eating behaviors during training and weight control periods by weight control level

Total score for eating behaviors during training period (possible score: 16-48) was 30.3, which was 63.1 out of 100 . There was no significant difference in total score of eating behaviors during training period between the HWL and NWL groups (Table 5). When each eating behavior in both groups was examined, percentages of those who showed desirable eating behaviors during training period such as eating meals regularly, eating an adequate amount of meals, and adequate consumption of foods in food groups were low. None of the
16 eating behaviors during training period were significantly different between the HWL and NWL groups (Table 5).

Total score for eating behaviors during weight control period (possible score: 16-48) was 28.4, which was 59.2 out of 100 . Total score for eating behaviors of the HWL group was significantly lower than that of the NWL group when considering covariates of age, $\mathrm{BMl}$, and sports type ( 27.8 vs. $30.2, P<0.001)$. Twelve out of the 16 eating behaviors during weight control period were significantly different by weight control level (Table 6). Percentages of those who performed desirable behaviors (3-7 days a week before competition) were significantly lower

Table 5. Eating behaviors of subjects during training period by weight control level

\begin{tabular}{|c|c|c|c|c|c|c|c|}
\hline \multirow{3}{*}{$\begin{array}{c}\text { Variables } \\
\text { (days/week) }\end{array}$} & \multicolumn{6}{|c|}{ 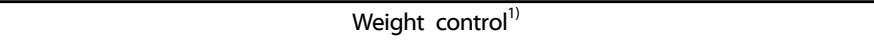 } & \multirow{3}{*}{$x^{25)}$} \\
\hline & \multicolumn{3}{|c|}{ NWL $(n=41)$} & \multicolumn{3}{|c|}{ HWL $(n=110)$} & \\
\hline & $0-2$ & $3-5$ & $6-7$ & $0-2$ & 3-5 & $6-7$ & \\
\hline \multicolumn{8}{|l|}{ Desirable eating behaviors during training period } \\
\hline 1. Eat three meals regularly. & $11(26.8)^{2)}$ & $23(56.1)$ & $7(17.1)$ & $33(30.0)$ & $62(56.4)$ & $15(13.6)$ & 0.3 \\
\hline 2. Eat adequate amount of meals. & $7(17.1)$ & $24(58.5)$ & $10(24.4)$ & $19(17.3)$ & $66(60.0)$ & $25(22.7)$ & 0.0 \\
\hline 3. Eat a variety of foods at each meal. & $10(24.4)$ & $23(56.1)$ & $8(19.5)$ & $22(20.0)$ & $63(57.3)$ & $25(22.7)$ & 0.4 \\
\hline 4. Eat breakfast & $24(58.5)$ & $10(24.4)$ & $7(17.1)$ & $56(50.9)$ & $42(38.2)$ & $12(10.9)$ & 2.9 \\
\hline 5. Eat grains three times a day. & $10(24.4)$ & $21(51.2)$ & $10(24.4)$ & $36(32.7)$ & $56(50.9)$ & $18(16.4)$ & 1.7 \\
\hline 6. Eat 1-2 protein foods or more at meals. & $14(34.1)$ & $17(41.5)$ & $10(24.4)$ & $30(27.3)$ & $56(50.9)$ & $24(21.8)$ & 1.1 \\
\hline 7. Eat $1-2$ vegetables or more at meals. & $7(17.1)$ & $25(61.0)$ & $9(22.0)$ & $20(18.2)$ & $60(54.5)$ & $30(27.3)$ & 0.6 \\
\hline 8. Eat dairy products once or more a day. & $13(31.7)$ & $23(56.1)$ & $5(12.2)$ & $26(23.6)$ & $58(52.7)$ & $26(23.6)$ & 2.7 \\
\hline 9. Drink about eight glasses of water a day. & $7(17.1)$ & $11(26.8)$ & $23(56.1)$ & $24(21.8)$ & $43(39.1)$ & $43(39.1)$ & 3.6 \\
\hline \multicolumn{8}{|l|}{ Eating foods high in $\omega 3$ fatty acids during training period } \\
\hline 10. Eat fish high in $\omega 3$ fatty acids once or more a day. & $36(87.8)$ & $5(12.2)$ & $0(0.0)$ & $86(78.2)$ & $20(18.2)$ & $4(3.6)$ & 2.5 \\
\hline 11. Eat a handful of nuts once a day. & $36(87.8)$ & $4(9.8)$ & $1(2.4)$ & $89(80.9)$ & $15(13.6)$ & $6(5.5)$ & 1.1 \\
\hline \multicolumn{8}{|l|}{ Undesirable eating behaviors during training period } \\
\hline 12. Eat fatty foods. & $8(19.5)$ & $30(73.2)$ & $3(7.3)$ & $26(23.6)$ & $74(67.3)$ & $10(9.1)$ & 0.5 \\
\hline 13. Eat spicy and salty foods. & $7(17.1)$ & $29(70.7)$ & $5(12.2)$ & $23(20.9)$ & $74(67.3)$ & $13(11.8)$ & 0.3 \\
\hline 14. Eat sweet foods. & $6(14.6)$ & $24(58.5)$ & $11(26.8)$ & $13(11.8)$ & $67(60.9)$ & $30(27.3)$ & 0.2 \\
\hline 15. Eat processed foods or instant foods. & $5(12.2)$ & $26(63.4)$ & $10(24.4)$ & $25(22.7)$ & $67(60.9)$ & $18(16.4)$ & 2.7 \\
\hline 16. Drink at least a cup of caffeinated beverages a day. & $14(34.1)$ & $20(48.8)$ & $7(17.1)$ & $43(39.1)$ & $45(40.9)$ & $22(20.0)$ & 0.8 \\
\hline Total score of eating behaviors ${ }^{3)}$ & & $30.0 \pm 4.4^{4)}$ & & & $30.4 \pm 3.6$ & & $1.8^{6)}$ \\
\hline \multicolumn{8}{|c|}{$\begin{array}{l}\text { 1) } \mathrm{NWL}, \leq 5 \% \text { loss of body weight; HWL, }>5 \% \text { loss of body weight. } \\
\text { 2) } \mathrm{N}(\%) \\
\text { 3) } \text { Possible total score: } 16-48 \text {. To calculate the total score, each question was coded from } 1 \text { (0-2days/week) to } 3 \text { (6- } 7 \text { days/week), and the items from } 12 \text { to } 16 \text { were scored } \\
\text { reversely. The higher total score means the more desirable eating behaviors. } \\
{ }^{4)} \text { Mean } \pm \mathrm{SD} \\
\text { 5) } \chi^{2} \text { value by } \chi^{2} \text {-test } \\
\text { 6) value by ANCOVA with covariates of age, BMl and sports type. }\end{array}$} \\
\hline
\end{tabular}

Table 6. Eating behaviors of subjects during weight control period by weight control level

\begin{tabular}{|c|c|c|c|c|c|c|c|}
\hline \multirow{3}{*}{$\begin{array}{c}\text { Variables } \\
\text { (days/week) }\end{array}$} & \multicolumn{6}{|c|}{ Weight control $^{1)}$} & \multirow{3}{*}{$x^{25)}$} \\
\hline & \multicolumn{3}{|c|}{ NWL $(n=41)$} & \multicolumn{3}{|c|}{ HWL $(n=110)$} & \\
\hline & $0-2$ & 3-5 & $6-7$ & $0-2$ & 3-5 & $6-7$ & \\
\hline \multicolumn{8}{|c|}{ Desirable eating behaviors during weight control period } \\
\hline 1. Eat three meals regularly. & $16(39.0)^{2)}$ & $24(58.5)$ & $1(2.4)$ & $82(74.5)$ & $26(23.6)$ & $2(1.8)$ & $16.9^{* * *}$ \\
\hline 2. Eat adequate amount of meals. & $10(24.4)$ & $24(58.5)$ & $7(17.1)$ & $80(72.7)$ & $25(22.7)$ & $5(4.5)$ & $29.4^{* * *}$ \\
\hline 3. Eat a variety of foods at each meal. & $16(39.0)$ & $21(51.2)$ & $4(9.8)$ & 81 (73.6) & $27(24.5)$ & $2(1.8)$ & $17.0^{* * *}$ \\
\hline 4. Eat breakfast & $23(56.1)$ & $16(39.0)$ & $2(4.9)$ & $90(81.8)$ & $17(15.5)$ & $3(2.7)$ & $10.7^{* *}$ \\
\hline 5. Eat grains three times a day. & $13(31.7)$ & $22(53.7)$ & $6(14.6)$ & $84(76.4)$ & $24(21.8)$ & $2(1.8)$ & $28.5^{* * *}$ \\
\hline 6. Eat $1-2$ protein foods or more at meals. & $20(48.8)$ & $14(34.1)$ & $7(17.1)$ & $85(77.3)$ & $24(21.8)$ & $1(0.9)$ & $20.0^{* * *}$ \\
\hline 7. Eat 1-2 vegetables or more at meals. & 15 (36.6) & $21(51.2)$ & $5(12.2)$ & $71(64.5)$ & $35(31.8)$ & 4 (3.6) & $10.8^{* *}$ \\
\hline 8. Eat dairy products once or more a day. & $17(41.5)$ & $20(48.8)$ & $4(9.8)$ & $56(50.9)$ & $42(38.2)$ & $12(10.9)$ & 1.4 \\
\hline 9. Drink about eight glasses of water a day. & $13(31.7)$ & $10(24.4)$ & $18(43.9)$ & $68(61.8)$ & $25(22.7)$ & $17(15.5)$ & $15.5^{* * *}$ \\
\hline
\end{tabular}




\begin{tabular}{|c|c|c|c|c|c|c|c|}
\hline \multirow{3}{*}{$\begin{array}{c}\text { Variables } \\
\text { (days/week) }\end{array}$} & \multicolumn{6}{|c|}{ Weight control $^{1)}$} & \multirow{3}{*}{$\chi^{25)}$} \\
\hline & \multicolumn{3}{|c|}{ NWL $(n=41)$} & \multicolumn{3}{|c|}{ HWL $(n=110)$} & \\
\hline & $0-2$ & 3-5 & $6-7$ & $0-2$ & $3-5$ & $6-7$ & \\
\hline \multicolumn{8}{|l|}{ Eating foods high in $\omega 3$ fatty acids during training period } \\
\hline 10. Eat fish high in $\omega 3$ fatty acids once or more a day. & $36(87.8)$ & $4(9.8)$ & $1(2.4)$ & $101(91.8)$ & $7(6.4)$ & $2(1.8)$ & 0.6 \\
\hline 11. Eat a handful of nuts once a day. & $36(87.8)$ & $4(9.8)$ & $1(2.4)$ & $93(84.5)$ & $16(14.5)$ & $1(0.9)$ & 1.1 \\
\hline \multicolumn{8}{|l|}{ Undesirable eating behaviors during weight control period } \\
\hline 12. Eat fatty foods. & $23(56.1)$ & $17(41.5)$ & $1(2.4)$ & $92(83.6)$ & $17(15.5)$ & $1(0.9)$ & $12.5^{* *}$ \\
\hline 13. Eat spicy and salty foods. & $23(56.1)$ & $17(41.5)$ & $1(2.4)$ & $91(82.7)$ & $16(14.5)$ & $3(2.7)$ & $12.7^{* *}$ \\
\hline 14. Eat sweet foods. & $16(39.0)$ & $20(48.8)$ & $5(12.2)$ & $62(56.4)$ & $39(35.5)$ & $9(8.2)$ & 3.6 \\
\hline 15. Eat processed foods or instant foods. & $20(48.8)$ & $18(43.9)$ & $3(7.3)$ & $78(70.9)$ & $26(23.6)$ & $6(5.5)$ & $6.6^{*}$ \\
\hline 16. Drink at least a cup of caffeinated beverages a day. & $20(48.8)$ & $15(36.6)$ & $6(14.6)$ & $78(70.9)$ & $24(21.8)$ & $8(7.3)$ & $6.5^{*}$ \\
\hline Total score of eating behaviors ${ }^{3)}$ & & $30.2 \pm 3.9^{4)}$ & & & $27.8 \pm 2.8$ & & $15.6^{* * * 6)}$ \\
\hline \multicolumn{8}{|c|}{$\begin{array}{l}{ }^{*} P<0.05,{ }^{* *} P<0.01,{ }^{* *} P<0.001 \\
\text { 1) } \mathrm{NWL}, \leq 5 \% \text { loss of body weight; HWL, }>5 \% \text { loss of body weight. } \\
\text { 2) } \mathrm{N}(\%) \\
\text { 3) } \text { Possible total score: } 16-48 \text {. To calculate the total score, each question was coded from } 1 \text { (0-2days/week) to } 3 \text { (6-7 days/week), and the items from } 12 \text { to } 16 \text { were scored } \\
\text { reversely. The higher total score means the more desirable eating behaviors. } \\
\text { 4) Mean } \pm \mathrm{SD} \\
{ }^{5)} \chi^{2} \text { value by } \chi^{2} \text {-test } \\
{ }^{6} \mathrm{~F} \text { value by ANCOVA with covariates of age, BMl and sports type. }\end{array}$} \\
\hline
\end{tabular}

in the HWL group than the NWL group. These differences were noted in behaviors such as 'eating adequate amount of meals' $(P<0.001)$, 'eating grains three times a day' $(P<0.001)$, 'eating three meals regularly' $(P<0.001)$, 'eating a variety of foods at meals' $(P<0.001)$, 'eating protein foods at meals' $(P<0.001)$, 'drinking about eight glasses of water a day' $(P<0.001)$, 'having breakfast' $(P<0.01)$, and 'eating vegetables at meals' $(P<0.01)$. In contrast, percentages of those who performed undesirable eating behaviors infrequently ( $0-2$ days a week before competition) were significantly higher in the HWL group than the NWL group. More specifically, $83.6 \%$ and $82.7 \%$ of the HWL group consumed fatty foods and spicy/salty foods infrequently, respectively, compared to $56.1 \%$ of the NWL group $(P<0.01)$. Those who ate processed foods/instant foods and caffeinated beverages infrequently were $70.9 \%$ in the $\mathrm{HWL}$ group and $48.8 \%$ in the NWL group $(P<0.05)$.

Association of beliefs, self-efficacy, and eating behaviors to weight control level

Multiple logistic regression results at the total score level of variables are shown in Table 7. After adjusting for demographic variables of age, BMI, and sports type, self-efficacy regarding eating behaviors and weight control $(\mathrm{OR}=0.846,95 \% \mathrm{Cl}: 0.730$,
0.980) as well as eating behaviors during weight control period $(\mathrm{OR}=0.731,95 \% \mathrm{Cl}: 0.620,0.863)$ were found to be associated with a significantly decreased risk of being in the HWL group. In contrast, eating behaviors during training period $(\mathrm{OR}=1.285$, $95 \% \mathrm{Cl}: 1.112,1.485)$ were related to a significantly increased risk of being in the HWL group.

Based on the factor analysis, two subscales of beliefs, a self-efficacy scale, and three subscales of eating behaviors during training period and weight control period, respectively, were obtained. These included beliefs regarding advantages of weight control (three items, Cronbach's $a=0.66$ ), beliefs regarding disadvantages of weight control (five items, $a=0.73$ ), self-efficacy (five items, $a=0.71$ ), desirable eating behaviors (nine items, $a=0.78$ for training period, $a=0.84$ for weight control period,), undesirable eating behaviors (five items, $a=$ 0.70 for training period, $a=0.79$ for weight control period), and eating foods high in $\omega 3$ fatty acids (two items, $a=0.68$ for training period, $a=0.63$ for weight control period). Table 8 shows multiple logistic regression results at subscale levels. After controlling for age, BMl, and sports type, self-efficacy regarding eating behaviors and weight control $(O R=0.847,95 \%$ Cl: $0.720,0.996)$ as well as desirable eating behaviors during weight control period ( $\mathrm{OR}=0.688,95 \% \mathrm{Cl}$ : $0.564,0.840$ ) were

Table 7. Multiple logistic regression for high weight loss on beliefs, self-efficacy, and eating behaviors

\begin{tabular}{|c|c|c|}
\hline Variables & OR $(95 \% \mathrm{Cl})$ & $P$-value \\
\hline Beliefs regarding rapid weight control ${ }^{11)}$ & $0.965(0.874,1.066)$ & 0.488 \\
\hline Self-efficacy ${ }^{2)}$ & $0.846(0.730,0.980)$ & 0.026 \\
\hline Eating behaviors during training period ${ }^{3)}$ & $1.285(1.112,1.485)$ & 0.001 \\
\hline Eating behaviors during weight control period ${ }^{4)}$ & $0.731(0.620,0.863)$ & 0.000 \\
\hline
\end{tabular}

Multiple logistic regression for weight loss while controlling for age, BMl and sports type.

1) Summated score of eight items. Items of disadvantages of rapid weight loss were coded reversely.

2) Summated score of five items

3) Summated score of 16 items. Items of undesirable eating behaviors during training period (five items) were coded reversely.

4) Summated score of 16 items. Items of undesirable eating behaviors during weight control period (five items) were coded reversely. 
Table 8. Multiple logistic regression for high weight loss on subscales of beliefs, self-efficacy, and eating behaviors

\begin{tabular}{|c|c|c|}
\hline Variables & OR $(95 \% \mathrm{Cl})$ & $P$-value \\
\hline Beliefs regarding advantages of weight control $^{1)}$ & $1.196(0.938,1.525)$ & 0.148 \\
\hline Beliefs regarding disadvantages of weight control $^{2)}$ & $1.114(0.950,1.307)$ & 0.185 \\
\hline Self-efficacy ${ }^{3)}$ & $0.847(0.720,0.996)$ & 0.045 \\
\hline Desirable eating behaviors during training period ${ }^{4)}$ & $1.202(1.016,1.421)$ & 0.032 \\
\hline Undesirable eating behaviors during training period ${ }^{5)}$ & $0.792(0.589,1.066)$ & 0.125 \\
\hline Eating foods high in $\omega 3$ fatty acids during training period ${ }^{6)}$ & $2.200(0.954,5.076)$ & 0.065 \\
\hline Desirable eating behaviors during weight control period ${ }^{4)}$ & $0.688(0.564,0.840)$ & 0.000 \\
\hline Undesirable eating behaviors during weight control period ${ }^{5)}$ & $1.102(0.836,1.453)$ & 0.492 \\
\hline Eating foods high in $\omega 3$ fatty acids during weight control period ${ }^{6}$ & $0.948(0.397,2.263)$ & 0.904 \\
\hline
\end{tabular}

Multiple logistic regression for weight loss while controlling for age, BMl and sports type.

1) Summated score of three items

2) Summated score of five items

3) Summated score of five items

4) Summated score of nine items in each period (training period or weight control period)

5) Summated score of five items in each period

6) Summated score of two items in each period

associated with a significantly decreased risk of being in the HWL group. Desirable eating behaviors during training period $(\mathrm{OR}=1.202,95 \% \mathrm{Cl}: 1.016,1.421)$ were associated with a significantly increased risk of being in the HWL group.

\section{DISCUSSION}

This study examined whether or not weight control practices, beliefs, self-efficacy, and eating behaviors during training period and weight control period differ by weight control level in college weight class athletes. Subjects showed an average of $7.2 \%$ reduction in body weight when they tried weight control (not shown in Tables), and $72.8 \%$ of athletes reduced more than $5 \%$ of their body weight. Similarly, Kim et al. [21] found that weight-classed athletes lost $6.5 \%$ of their body weight. Previous studies have reported that weight class athletes showed an average body weight reduction of $2.5 \%-5.4 \%[6,11,12]$, which is lower than that of the current study. In a study on adolescent weight class athletes, Park et al. [34] reported that an $8 \%$ body weight reduction significantly decreased cardiovascular function capacity while a $3 \%$ body weight reduction did not, suggesting the optimal level of weight reduction. Similar to a previous study [8], it seems that subjects tried weight control before each competition, based on the finding that subjects participated in competitions 5.3 times and attempted weight control 5.1 times per year, on average. Subjects lost an average of $5.0 \mathrm{~kg}$ when they attempted weight control, and the magnitude of weight loss was significantly greater in the HWL than the NWL group $(P<0.001)$. Similarly, Brito et al. [11] reported that judo athletes reduced an average of $5.6 \mathrm{~kg}$ during the competitive week.

Subjects began weight control at the age of 15.7 years, which is similar to that reported in previous studies (14.0-16.5 years) $[5,11,21]$. Athletes in the HWL and NWL groups practiced weight control for an average of 12.6 days and 7.3 days before competition, respectively $(P<0.001)$, suggesting that weight loss was performed within a short time. Lee et al. [35] found that $57.5 \%$ of adolescent taekwondo athletes tried weight loss within 1 week before competition, whereas only $16 \%$ of them reduced weight gradually over 2-3 weeks before competition.
As for methods of weight control, a higher percentage of subjects in the HWL than the NWL group used inappropriate weight control methods such as fasting, restricting fluid intake, and saunas. Consistently, previous studies have reported that weight class athletes excessively performed weight loss using methods such as skipping meals, restricting fluids, training with plastic suits, and saunas $[6,11,21,35]$. Rapid and continuous weight loss in weight class athletes might cause malnutrition, loss of minerals due to dehydration, decreases in athletic performance, and physical injuries $[4,36]$. Inappropriate use of weight control methods might be related to dissatisfaction with weight control methods in the HWL group. Study results show that only $19.2 \%$ of athletes received education regarding weight control, which is lower than that reported in previous studies $[21,35]$. Results regarding weight control practices have suggested that education regarding appropriate weight control in weight class athletes is urgently needed.

The significant association between beliefs regarding rapid weight control and weight control level disappeared in the multiple logistic regression models, suggesting that beliefs regarding rapid weight control might be less important in explaining weight control level than other factors such as self-efficacy and eating behaviors during weight control period. When examined by each belief item, athletes in the HWL group agreed more strongly on the disadvantages of rapid weight loss than the NWL group. This suggests that athletes in the HWL group tried to lose weight even though they acknowledged the disadvantages and adverse effects of rapid weight loss. This might be due to the perception that rapid weight loss and competing at a lower weight class resultantly increases the possibility of winning. Therefore, it is urgent to educate college weight class athletes on the reality that rapid weight loss does not necessarily lead to better athletic performance and winning [37], in addition to the adverse effects of rapid weight loss. Instead, advantages and tips related to proper weight control through gradual diet and exercise need to be emphasized.

Self-efficacy was found to be important in differentiating the HWL and NWL groups. The odds ratio for HWL was 0.85 with unit increase in self-efficacy score. It seems that athletes in this 
study were not confident in maintaining adequate eating behaviors related to weight control, based on their total score for self-efficacy (59.6 out of 100). In addition, subjects in the HWL group compared to the NWL group were less confident regarding specific self-efficacy (controlling excessive overeating/ binge eating after matches and making weight within their weight class through gradual dieting and exercise) as well as total self-efficacy. These results support the notion that self-efficacy is important in performing a behavior (e.g., adequate weight control). Consistently, Kang \& Kim [38] observed that self-efficacy was a strong predictor of older adults' physical activity, followed by perceived exercise benefits, family support, and physical environment. In a study on college female athletes, dietary self-efficacy and nutrient intakes (e.g., carbohydrate, fiber) were significantly elevated after nutrition education programs focused on self-efficacy [39]. These study results imply that education programs need to focus on increasing selfefficacy by providing strategies to adopt desirable eating behaviors (e.g., methods for gradual adoption of proper eating habit, methods for step-by-step dieting and exercise, resisting temptations to overeat after matches, etc.). Self-efficacy might be elevated through methods such as providing opportunities to perform a behavior, indirect experience (e.g., modeling), persuasion, and praise [18].

This study shows that eating behaviors of college weight class athletes were generally inadequate during training period as well as pre-competition period. Based on multiple logistic regression analyses, it was shown that eating behaviors during training period as well as weight control period were associated with a specific weight control group (HWL vs. NWL). The odds for HWL increased with an increased score for eating behaviors during training period, whereas the odds for HWL were reduced with an increased score for eating behaviors during weight control period. In addition, it seems that the association of eating behaviors to weight control group was mainly due to differences in desirable eating behaviors during each period (e.g., eating meals regularly, eating adequate amounts, eating a variety of foods, and adequate consumption of foods in food groups) based on the finding of subscale level analysis of the multiple logistic regression.

Athletes in the HWL group practiced inadequate eating behaviors, especially during weight control periods. This might be due to excessive dieting or fasting to make weight. Consistent with the current study, previous studies have reported the undesirable eating behaviors of weight-classed athletes $[7,10,26]$. Specifically, intakes of energy and nutrients of weight-classed athletes were significantly reduced before competition and increased after competition [7]. Fleming \& Costarelli [10] also reported that weight class athletes consumed a suboptimal diet both at ordinary times and during pre-competition, and intakes of energy, carbohydrates, calcium, and sodium during precompetition were significantly lower than that at ordinary times. In a study on adolescent taekwondo athletes, Jung et al. [40] reported that consumption of foods such as protein-rich foods and processed foods was reduced during training period before competition compared to usual training period. Restriction of food intakes during the weight control period might lead to overconsumption of foods after competition, causing weight cycling commonly found in weight class athletes.

The limitation of this study is that the study results are based on a convenient sample of college weight class athletes in Gyeong-gi Province, Korea. Therefore, the study findings might not be generalized to different groups of weight class athletes. In future research, factors related to weight control might be broadened to include environmental influences.

In summary, this study shows that college weight class athletes practiced weight loss inappropriately. Use of weight control methods, such as restricting fluid intake and using plastic suits and saunas, were more prevalent in the HWL compared to NWL group. Self-efficacy, eating behaviors during training period, as well as eating behaviors during weight control period were also significantly associated with weight control group. Based on this study, nutrition education for college weight class athletes should be provided to make weight by using appropriate weight control methods in collaboration with tailored, gradual weight loss approaches. Further, information regarding the adverse effects of rapid weight loss and use of inappropriate weight control methods is needed. Education programs also need to include strategies to increase self-efficacy as well as methods to alter eating behaviors before competition, especially for those who try lose weight rapidly.

\section{ACKNOWLEDGEMENTS}

We are grateful to the weight class athletes who participated in the survey and coaches who cooperated to conduct the survey.

\section{CONFLICT OF INTEREST}

The authors declare no potential conflicts of interest.

\section{ORCID}

Ji Seon Lee: http://orcid.org/0000-0001-7592-8565

Seong Suk Cho: http://orcid.org/0000-0002-8938-0147

Kyung Won Kim: http://orcid.org/0000-0002-6569-9006

\section{REFERENCES}

1. Thomas DT, Erdman KA, Burke LM. Position of the academy of nutrition and dietetics, dietitians of Canada, and the American College of Sports Medicine: nutrition and athletic performance. J Acad Nutr Diet 2016;116:501-28.

2. Kim Ml. The review on nutritional management for competitive weight control athletes. J Coaching Dev 2005;7:3-12.

3. Langan-Evans C, Close GL, Morton JP. Making weight in combat sports. Strength Condit J 2011;33:25-39.

4. Franchini E, Brito CJ, Artioli GG. Weight loss in combat sports: physiological, psychological and performance effects. J Int Soc Sports Nutr 2012;9:52-7.

5. Steen SN, Brownell KD. Patterns of weight loss and regain in wrestlers: has the tradition changed? Med Sci Sports Exerc 1990;22: 762-8.

6. Artioli GG, Gualano B, Franchini E, Scagliusi FB, Takesian M, Fuchs 
M, Lancha AH Jr. Prevalence, magnitude, and methods of rapid weight loss among judo competitors. Med Sci Sports Exerc 2010;42: 436-42.

7. Son HS, Lee SJ. A study on the nutrient intakes of the taekwondo players before and after the games. J Taekwondo Sci 2009;2:43-9.

8. $\mathrm{Kim} \mathrm{JH}$, Choue RW. The study of nutrients intake, nutrition knowledge and weight loss patterns for 12 weeks pre-competition in college taekwondo players. Korean J Phys Educ 2004;43:473-84.

9. Shin MG, Kim HY, Park S, Park H. Analysis of nutrient intake and beverage intake status of male taekwondo players. Korean J Sport Sci 2010;21:919-28.

10. Fleming $\mathrm{S}$, Costarelli V. Nutrient intake and body composition in relation to making weight in young male taekwondo players. Nutr Food Sci 2007;37:358-66.

11. Brito CJ, Roas A FC, Brito I SS, Marins J CB, Córdova C, Franchini E. Methods of body mass reduction by combat sport athletes. Int J Sport Nutr Exerc Metab 2012;22:89-97.

12. Coufalová $\mathrm{K}$, Prokešová $\mathrm{E}$, Malý $\mathrm{T}$, Heller J. Body weight reduction in combat sports. Arch Budo 2013;9:267-72.

13. Oppliger RA, Case HS, Horswill CA, Landry GL, Shelter AC. American College of Sports Medicine position stand. Weight loss in wrestlers. Med Sci Sports Exerc 1996;28:ix-xii.

14. Fogelholm M. Effects of bodyweight reduction on sports performance. Sports Med 1994;18:249-67.

15. Khodaee M, Olewinski L, Shadgan B, Kiningham RR. Rapid weight loss in sports with weight classes. Curr Sports Med Rep 2015;14: 435-41.

16. Montano DE, Kasprzyk D. Theory of reasoned action, theory of planned behavior, and the integrated behavioral model. In: Glanz K, Rimer BK, Viswanath K, editors. Health behavior: theory, research, and practice. 5th ed. San Francisco: Jossey-Bass; 2015. p.95-124.

17. Kelder SH, Hoelscher D, Perry CL. How individuals, environments, and health behaviors interact. In: Glanz K, Rimer BK, Viswanath K, editors. Health behavior: theory, research, and practice. 5th ed. San Francisco: Jossey-Bass; 2015. p.159-82.

18. Contento IR. Nutrition education: linking research, theory, and practice. 3rd ed. Burlington: Jones \& Bartlett Learning; 2015. p.108-24, 135-48.

19. Kim YS, Kim YI. The structural relationship among achievement goal orientation, intrinsic motivation, self-efficacy and exercise adherence in university taekwondo players. J Sport Leis Stud 2011;46:593-606.

20. Lee GO. Self-regulation, self-efficacy, and exercise adherence of physical education middle and high school students. J Korean Soc Study Phys Educ 2012;17:105-16.

21. Kim CH, Kim C, Ji JW, Pho JH, Oh HS, Choi YE. Weight loss patterns of weight-classed athletes in Korea: a descriptive study. Korean J Sports Med 2001;19:49-61.

22. Kim YS, Kim GJ, Shin JT, Kim HY, Lim GW. Application and development of nutritional guidelines for physical conditions to pass weight in judo players. Seoul: Korea Institute of Sport Science; 2009.
23. Seo GR, Kwon SY. Exploration of male weight class athletes' experiences of eating disorder. Korean J Phys Educ 2013;52:83-94.

24. Kim MJ, Kim KW. Nutrition knowledge, outcome expectations, self-efficacy, and eating behaviors by calcium intake level in Korean female college students. Nutr Res Pract 2015;9:530-8.

25. Kim JY, Lee JS, Cho SS, Park H, Kim KW. Nutrient intakes of male college combat sport athletes by weight control status. Korean J Community Nutr 2017;22:495-506.

26. Fleming $S$, Costarelli V. Eating behaviours and general practices used by taekwondo players in order to make weight before competition. Nutr Food Sci 2009;39:16-23.

27. Chun KY, Oh IS. The effect of different weight reduction period on cell blood count and anaerobic capacity in boxing athletics. Korean J Sports Sci 2003;12:589-97.

28. Chun YS, Lee KE, Back NS, Kim MS, Lee KW, Kim SY. The nutrition indigestion of the high school judo players in accordance to their weight, before and after the competition. J Korean Alliance Martial Arts 2010;12:299-310.

29. Lindeman AK. Self-esteem: its application to eating disorders and athletes. Int J Sport Nutr 1994;4:237-52.

30. Seok HK, Her ES. The weight control-related attitudes, dietary self-efficacy and eating behaviors according to the perception of eating disorder and obesity index in dancing major high school girls. Korean J Community Nutr 2009;14:383-91.

31. Lee KY, Lee MC. The diet programs for elite athletes. J Coaching Dev 2003;5:89-101.

32. Sung $\mathrm{H}$, Chang KJ. A survey on self-reported health and eating habit of elite female adolescent athletes in Incheon by sport type. J Korean Soc Food Sci Nutr 2005;34:366-73.

33. Rhyu HS. Effects of short term weight loss on physiological variables in weight category sports. Korean J Sport Sci 2016;27:209-19.

34. Park MK, Sung JM, Kang HS. Research about establishment of the most suitable weight reduction level. Exerc Sci 2009;18:143-50.

35. Lee JK, Kim C, Kim CH, Kwon YW, Kim JH. Weight loss patterns of the young taekwondo athletes in Korea: a descriptive study. Korean J Sport Sci 2002;13:81-90.

36. Cho YK. A study on weight control players' eating behavior. Korea Sports Res 2003; 14:395-406.

37. Kazemi M, Rahman A, De Ciantis M. Weight cycling in adolescent taekwondo athletes. J Can Chiropr Assoc 2011;55:318-24.

38. Kang SJ, Kim YH. The effect of individual, social, and physical environment variables on older adults' physical activity. Korean $J$ Sport Psyc 2011;22:113-24.

39. Abood DA, Black DR, Birnbaum RD. Nutrition education intervention for college female athletes. J Nutr Educ Behav 2004;36:135-9.

40. Jung KA, Hwang SH, Kim C, Lee JK, Chang YK. An evaluation of the nutritional status and the desirable time and period for dietary record in male high school taekwondo athletes. Korean J Nutr 2002;35:237-49. 